\title{
Bitvectors with runs and the successor/predecessor problem
}

\author{
Adrián Gómez-Brandón \\ Universidade da Coruña \\ Laboratorio de Bases de Datos, CITIC \\ A Coruña, Spain \\ adrian.gbrandon@udc.es
}

\begin{abstract}
The successor and predecessor problem consists of obtaining the closest value in a set of integers, greater/smaller than a given value. This problem has interesting applications, like the intersection of inverted lists. It can be easily modeled by using a bitvector of size $n$ and its operations rank and select. However, there is a practical approach [1], which keeps the best theoretical bounds, and allows to solve successor and predecessor more efficiently. Based on that technique, we designed a novel compact data structure for bitvectors with $k$ runs that achieves access, rank, and successor/predecessor in $O(1)$ time by consuming space $O(\sqrt{k n})$ bits. In practice, it obtains a compression ratio of $0.04 \%-26.33 \%$ when the runs are larger than 100, and becomes the fastest technique, which considers compressibility, in successor/predecessor queries. Besides, we present a recursive variant of our structure, which tends to $O(k)$ bits and takes $O\left(\log \frac{n}{k}\right)$ time.
\end{abstract}

\section{Introduction}

One of the main computational tasks in a search engine is to look for those documents that contain a set of words. In order to speed up that search, those engines use inverted lists. Each inverted list corresponds to a word and stores as an increasing sequence the document identifiers of the documents where that word occurs. Most of the time, the query received by a search engine carries more than one word, to know where all the words appear together, the search engine needs to intersect various inverted lists. The optimal intersection of two lists can be easily solved by iterating over both of them in alternate form [2]. In each iteration, the search engine looks for a value in the second list, $v_{2}$, equal to or higher than the value from the first list, $v_{1}$. If they are identical, $v_{1}$ is part of the solution and iterates to the next value in the first list. Otherwise, the iterator of the first list skips those values lower than $v_{2}$. Therefore, it needs an efficient mechanism that can find an equal or higher value in the other list, which is known as the successor problem.

Let us formalize the successor and predecessor problem, considering a set of integers $S=\left\{x_{1}<x_{2}<\cdots<x_{m}\right\}$, the successor $\left(\operatorname{succ}(x)=x_{i}\right)$ of a given value $x$ returns the minimum value $x_{i} \geq x$ of $S$. Analogously, the predecessor of $x\left(\operatorname{pred}(x)=x_{i}\right)$ returns the maximum value $x_{i} \leq x$ of $S$. Assuming $n=x_{m}$ and $m=|S|$, both problems can be modeled by using a bitvector $B[1, n]$ which contains $m$ 1s located

Funded in part by European Unions Horizon 2020 Marie Skłodowska-Curie grant agreement No 690941; MINECO (PGE and FEDER) [TIN2016-78011-C4-1-R]; Xunta de Galicia (co-founded with FEDER) [ED431G/01; ED431C 2017/58; IN852A 2018/14], Xunta de Galicia (co-founded with GAIN) [IN848D-2017-2350417] and FPU Program [FPU16/02914] 
at positions $x_{i}$ for all $1 \leq i \leq m$, and solved in $O(1)$ time with the two classical operations on bitvectors: rank and select [3-6].

In some scenarios, the bitvector $B$ can contain the set bits clustered together in $k$ runs; hence $B$ contains $k$ runs of 1 s and $k \pm 1$ runs of 0 s. There is a structure, oz-vector [1], which compresses the bitvectors exploiting its runs. The oz-vector transforms the input bitvector into two sparse bitvectors $O$ and $Z$, which mark the lengths of the runs of $1 \mathrm{~s}$ and $0 \mathrm{~s}$, respectively. Since those bitvectors are sparse, they are very compressible, and the oz-vector can obtain good compression ratios in practice. However, for solving succ and pred, it requires $O(\log k)$ time. This study aims to propose a new structure, zombit-vector, which compresses bitvectors with runs and supports succ and pred in $O(1)$ time. zombit-vector splits the bitvector into blocks of fixed size $\beta$ and classifies them into three sets of blocks depending on their information: full of $0 \mathrm{~s}(\mathcal{Z})$, full of $1 \mathrm{~s}(\mathcal{O})$, and containing $1 \mathrm{~s}$ and $0 \mathrm{~s}(\mathcal{M})$. Representing this information needs $O\left(k \beta+\frac{n}{\beta}\right)+o(n)$ bits. As the optimal value of $\beta$ is $\sqrt{\frac{n}{k}}$, it can solve access, rank, succ, and pred in $O(1)$ time with $O(\sqrt{k n})$ bits. Besides, we present a variant which constructs a zombit-vector recursively. That recursive technique converges to $O(k)$ bits and can solve those operations in $O\left(\log \frac{n}{k}\right)$.

We compare our proposal with different compact data structures for bitvectors that can solve succ and pred efficiently. In the experimental evaluation, we can observe that zombit-vector obtains the best response times in all settings. It becomes $5-12$ times faster than our immediate competitor and occupies $0.04 \%-26.33 \%$ of the plain bitvector, when the mean length of runs is higher than 100 .

\section{Background}

A bitvector $B[1, n]$ is an array of bits whose size is $|B|=n$, and each position can acquire the two possible bit values, 0 and 1 . Usually, it is used to represent the values of $S=\left\{x_{1}, x_{2}, \ldots x_{m}\right\}$, a subset of an universe $\{1,2, \ldots n\}$, by setting $B\left[x_{i}\right]=1$ such that $x_{i} \in S$. Mainly, they support two operations: $\operatorname{rank}_{\alpha}(B, x)$, which returns the number of bits set to $\alpha$ within the interval $B[1, x]$; and $\operatorname{select}_{\alpha}(B, x)$, which returns the position of the $x$-th $\alpha$ value in $B$. In theory, both operations can be solved in $O(1)$ time by using additional structures which require $o(n)$ bits of extra-space [3-6].

Therefore, successor and predecessor problem can be modeled by setting $B\left[x_{i}\right]=1$ where $x_{i} \in S$ and both operations, with respect to a position $x$, can be solved as $\operatorname{succ}(B, x)=\operatorname{select}_{1}\left(B, \operatorname{rank}_{1}(B, x-1)+1\right)$ and $\operatorname{pred}(B, x)=\operatorname{select}_{1}\left(B, \operatorname{rank}_{1}(B, x)\right)$. Though both are $O(1)$ time, there is a more practical structure for succ/pred [1], which keeps the $o(n)$ extra-space and $O(1)$ time. In practice, it achieves less space and better response times than using rank, and then select. That structure is similar to the classical rank structure [3-6], but instead of storing the number of ones preceding

a position, it stores the location of the next/previous 1-bit. Since predecessor and successor are symmetrical, from this point on, we only refer to successor.

\section{Zero-order entropy}

Notice that, in a plain bitvector, we are using $n$ bits of space, which is the worst-case optimal, and achieves rank, select, and succ in $O(1)$ time with additional $o(n)$ space. 
However, a better lower bound of the representation of $S$ is $\mathcal{B}(n, m)=\left\lceil\log \left(\begin{array}{c}n \\ m\end{array}\right)\right\rceil$. In order to improve the worst-case optimal, in $[7,8]$ they propose techniques, which get $O(1)$ time in rank and select by using $\mathcal{B}(n, m)+o(n)$ bits. Consequently, succ can be solved in $O(1)$ time. The space result is approximately $n H_{0}$, where $H_{0}$ is the zero-order entropy of $B$. Some studies show new lower-bounds $[9,10]$ and confirm that the space of $[7,8]$ is almost the optimal.

\section{Sparse bitvectors}

Those bitvectors where $m \ll n$ are well-known as sparse bitvectors. In sparse bitvectors the extra $o(n)$ space can be huge, for this reason there are some proposals, like rec-rank and sd-array [11], focused on avoiding that dependency.

The first one splits $B$ into blocks of a given fixed size and classifies those partitions into two types: $Z$, full of 0 s, and $N Z$, the block contains at least one 1-bit. The kind of each block is stored into a contracted bitvector, $B_{c}$, and the $N Z$ blocks are grouped together by concatenating them preserving the order into an extracted bitvector, $B_{e}$. This process is repeated recursively over $B_{e}$ until it is not sparse. In total, it takes $\log \frac{n}{m}+m+o(n)$ bits and can solve rank, select, and succ in $O\left(\log \frac{n}{m}\right)$ time.

The sd-array defines a parameter $r=\left\lfloor\log \frac{n}{m}\right\rfloor$ and each value $S[i]$ is divided into the $r$ lowest bits $\left(l_{i}\right)$ and the $\lceil\log n\rceil-r$ most significant bits $\left(h_{i}\right)$. Notice that each $h_{i}$ covers an interval of values $\left[h_{i} \times 2^{r},\left(h_{i}+1\right) \times 2^{r}\right)$. With this information, the sd-array builds two elements, $L$ and $H$. $L$ is an array composed by each $l_{i}$, and $H$ is a bitvector that indicates in unary how many elements of $L$ are covered by all possible $h_{i}$. Since $L$ can be stored by using $m \log \frac{n}{m}$ bits, $H$ uses at most $3 m$ bits, and an additional structure of select; the required space is $m \log \frac{n}{m}+O(m)$ bits. It is able to solve select in $O(1)$ time, but rank and succ operations take $O\left(\log \frac{n}{m}\right)$.

Bitvectors with runs

Occasionally, a bitvector has the same distribution of 1s and 0s; however, both bits are clustered together forming runs. Since the number of 1s and 0s are similar, the zeroorder entropy cannot capture the compressibility of bitvectors with runs. Therefore, the previous techniques are not useful in this case.

Nevertheless, in [1] they propose a structure called oz-vector, which makes possible to use those previous techniques by transforming $B$ into two sparse bitvectors $(O$ and $Z$ ). The transformation consists in computing the length $\ell$ of every run of $1 \mathrm{~s}$ and 0 s, and storing those lengths with unary code $\left(10^{\ell}\right)$ in $O$ and $Z$, respectively. Consequently, the length of the first run of $1 \mathrm{~s}$ is the distance between the first and second 1-bit in $O$. By using the $s d$-array in $O$ and $Z$, the oz-vector uses $k \log \frac{2 n}{k}+O(k)$ bits and solves select in $O\left(\min \left(\log k, \log \frac{n}{k}\right)\right)$ and rank in $O(\log k)$. Therefore, succ can be solved in $O(\log k)$.

\section{Hybrid bitvectors}

As it is shown in the previous scenarios, we can find different types of bitvectors depending on its number of 1s or how clustered are those bits. In [12], the authors propose a structure (hybrid-vector) that can adapt its compression technique according to the features of its input. The hybrid-vector splits the input into blocks of a 


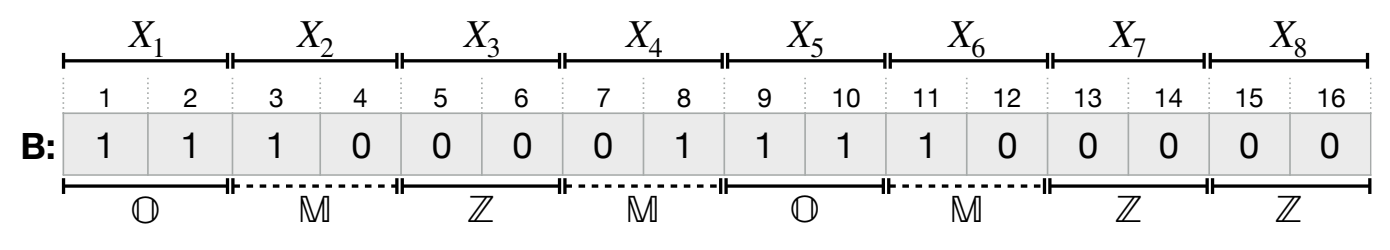

\begin{tabular}{|c|c|c|c|c|c|c|c|c|c|}
\hline \multirow{6}{*}{ zombit-vector: } & \multirow[b]{2}{*}{ U: } & 1 & 2 & 3 & 4 & 5 & 6 & 7 & 8 \\
\hline & & 1 & 0 & 1 & 0 & 1 & 0 & 1 & 1 \\
\hline & \multirow{3}{*}{ O: } & 1 & 2 & 3 & 4 & 5 & 6 & 7 & 8 \\
\hline & & 1 & 1 & 0 & 1 & 1 & 1 & 0 & 0 \\
\hline & & 1 & 2 & 3 & 4 & 5 & 6 & & \\
\hline & M: & 1 & 0 & 0 & 1 & 1 & 0 & & \\
\hline
\end{tabular}

Figure 1: Example of zombit-vector with $\beta=2$.

fixed size $b$, and each one is encoded individually. There are three possible types of block encoding: (i) minority bit positions, which stores only the positions of all 1s or 0s, the one which has fewer occurrences; (ii) run-length encoding, stores the length of every run; and (iii) plain, in case that the previous options are not satisfactory, the block is encoded in plain form. Over those blocks, in order to speed up rank and select, there is an auxiliary structure of super-blocks storing the accumulative rank. In the worst case it takes $n+o(n)$, but if it has $k$ runs and $m$ minority ones, it only uses $\min (k, m)\lceil\log b\rceil+o(n)$ bits. With this space, it achieves rank in $O(1)$, but select and succ require $O(\log n)$ time.

\section{zombit-vector}

Our proposal, zombit-vector is designed to compress bitvectors with runs and solve successor and predecessor operations in $O(1)$ time. The main idea is to divide the input into blocks in such a way that most of the blocks are uniform (all 0s or all 1s). With this approach, our structure only needs to store the information contained by non-uniform blocks.

\section{Structure}

Given a bitvector $B$ of size $|B|=n$ with $k$ runs of 1 s and $k \pm 1$ runs of 0 s, zombit-vector defines a size of block $\beta$ which splits $B$ into $\left\lceil\frac{n}{\beta}\right\rceil$ partitions, obtaining a set of blocks $\left\{X_{1}, X_{2}, \ldots, X_{\left\lceil\frac{n}{\beta}\right\rceil}\right\}$. Each block $X_{i}$ can be classified into three different sets of blocks depending on its data: uniform blocks full of $0 \mathrm{~s}(\mathcal{Z})$, uniform blocks full of $1 \mathrm{~s}(\mathcal{O})$, and mixed blocks, those which contain both bits $(\mathcal{M})$. As a consequence, the structure contains $u=|\{\mathcal{Z} \cup \mathcal{O}\}|$ uniform and $t=\left\lceil\frac{n}{\beta}\right\rceil-u$ mixed blocks. The classification of each block can be represented by using two plain bitvectors: $U$ and $O$. The bitvector $U\left[1,\left\lceil\frac{n}{\beta}\right\rceil\right]$ marks which $X_{i}$ is uniform by setting $U[i]=1$ when $X_{i} \in\{\mathcal{Z} \cup \mathcal{O}\}$. Then, 
we use the bitvector $O\left[1,\left\lceil\frac{n}{\beta}\right\rceil\right]$ to represent which block contains at least one 1-bit, it means $O[i]=1$ when $X_{i} \in\{\mathcal{O} \cup \mathcal{M}\}$. Additionally to this classification, we need to store the data of every mixed block. For that purpose, we use a bitvector $M[1, t \times \beta]$ which appends the information of each mixed block together, preserving the order in $B$. In total, we need $O\left(k \beta+\frac{n}{\beta}\right)$ bits, and since the optimal $\beta=\sqrt{\frac{n}{k}}$, we can reduce the space to $O(\sqrt{k n})$ bits.

Figure 1 shows an example of zombit-vector built over the bitvector $B$ with $\beta=2$. On top of Figure 1, $B$ is divided into 8 blocks $\left\{X_{1}, X_{2}, \ldots, X_{8}\right\}$. On the bottom of each block, we can observe the set which contains such block and solid or dashed lines, which distinguish uniform and mixed blocks, respectively. For instance, $X_{1} \in \mathcal{O}$ is uniform, thus it gets a solid line, but $X_{2} \in \mathcal{M}$ is mixed, and it is represented with a dashed line. As $X_{2}, X_{4}, X_{6}$ are the mixed blocks, in the bitvector $U$ of the zombitvector structure, all the bits are set to 1 except 2, 4 and 6 . Concerning the $O$ bitvector, it has only 0-bits at positions 3,7 , and 8 because $X_{3}, X_{7}, X_{8}$ belong to $\mathcal{Z}$. Finally, the mixed blocks are sequentially stored in the bitvector $M$, hence $M[1,2]$ corresponds with $X_{2}, M[3,4]$ with $X_{4}$ and $M[5,6]$ with $X_{6}$.

\section{Basic operators}

For a better explanation of the operations that can be solved in the zombit-vector structure, let us define two basic operators:

- $b e g_{i}$ and $e n d_{i}$ : given a position $i$, which represents the $i$-th block, it returns the first/last position, respectively, where the information of the $i$-th block is located. The result can be computed as $\operatorname{beg}_{i}=(i-1) \times \beta+1$ and $e n d_{i}=i \times \beta$.

- $\Delta_{i}$ : computes the distance of $i$ with respect to the first position of its block as $(i-1) \bmod \beta$. For example in Figure $1, \Delta_{8}=1$ because of $\beta=2$ the first position of its block is 7 , hence the distance is $8-7=1$.

\section{Access}

Access operation retrieves the value at a given position $i$. That information is stored on a block $X_{j}$, where $j=\left\lceil\frac{i}{\beta}\right\rceil$. If $X_{j}$ is uniform $(U[j]=1)$, all the values inside that block are identical. Since $O[j]$ indicates when a block contains at least 1-bit, the uniform block is full of 1 s when $O[j]=1$; otherwise, it is empty. Therefore, in the uniform case, access can be solved by returning $O[j]$. For instance, with $i=5$ the solution is in $X_{3}$, an uniform block $(U[3]=1)$, thus the algorithm returns $O[3]=0$.

Otherwise, when $X_{j}$ is mixed $(U[j]=0)$, the number of mixed blocks up to $j$ is computed as $q=\operatorname{rank}_{0}(U, j)$. Consequently, the value of $i$ is inside the $q$-th mixed block, specifically, $\Delta_{i}$ positions after the first position of that block. As the data of the $q$-th mixed block are at $M\left[b e g_{q}\right.$, end $\left.d_{q}\right]$, the value corresponds with $M\left[b e g_{q}+\Delta_{i}\right]$. For example, in Figure 1 to retrieve $B[7]$, the algorithm computes $j=4$ and checks $U[4] . X_{4}$ is a mixed block because $U[4]=0$, in particular, it is the second mixed block $\left(q=\operatorname{rank}_{0}(U, 4)=2\right)$. Hence, the algorithm returns $M\left[b_{e} g_{2}+\Delta_{7}\right]=M[3+0]=0$. As we can observe, in both cases, this operation can be solved in $O(1)$ time by using $o(n)$ bits of additional space for rank in plain bitvectors. 
Rank

$\operatorname{rank}_{1}(B, i)$ computes the number of $1 \mathrm{~s}$ in $B$ up to a position $i$ which belongs to a block $X_{j}$. In zombit-vector, the algorithm starts computing the number of 1 s preceding to $X_{j}$. Let us define $p^{\prime} / q^{\prime}$, as the number of uniform/mixed blocks previous to $X_{j}{ }^{1}$ Therefore, the uniform blocks include $b_{1}=p^{\prime}-\operatorname{rank}_{0}(O, j-1)$ blocks full of 1s. Since there are $b_{1}$ blocks with $\beta$ 1s each, and $q^{\prime}$ mixed blocks that accumulate $\operatorname{rank}_{1}\left(M\right.$, end $\left._{q^{\prime}}\right) 1 \mathrm{~s}$, the number of $1 \mathrm{~s}$ before $X_{j}$ is $r=b_{1} \times \beta+\operatorname{rank}_{1}\left(M, e n d_{q^{\prime}}\right)$.

After computing the number of 1 s previous to $X_{j}$, the algorithm updates $r$ depending on the number of $1 \mathrm{~s}$ in $X_{j}$. If $X_{j} \in \mathcal{Z}$, the block is absent of 1 s and the algorithm returns $r$. When $X_{j} \in \mathcal{O}$, the number of ones contained by $X_{j}$ up to $i$ must be considered. Since $X_{j}$ is full of $1 \mathrm{~s}$, thus it contains $\Delta_{i}+1$ extra $1 \mathrm{~s}$, the solution is $r+\Delta_{i}+1$. Otherwise, $X_{j}$ belongs to $\mathcal{M}$, which is the $\left(q^{\prime}+1\right)$-th mixed block. The number of ones up to $i$ inside $X_{j}$ is computed as $b=\operatorname{rank}_{1}\left(M, \operatorname{beg}_{\left(q^{\prime}+1\right)}+\Delta_{i}\right)-\operatorname{rank}_{1}\left(M, \operatorname{end}_{q^{\prime}}\right)$, thus the solution becomes $r+b$. Notice that, we can simplify that formula as $b_{1} \times \beta+\operatorname{rank}_{1}\left(M, \operatorname{beg}_{\left(q^{\prime}+1\right)}+\Delta_{i}\right)$, the number of 1 s previous to $X_{j}$ in those blocks full of $1 \mathrm{~s}$, and the number of $1 \mathrm{~s}$ inside the mixed blocks up to the queried position. For example, in Figure 1 for solving $\operatorname{rank}_{1}(B, 8)$ where $j=4$, the algorithm gets $p^{\prime}=2$, $q^{\prime}=1$, and $b_{1}=1 . X_{4}$ is a mixed block, hence the algorithm computes the first addend as $b_{1} \times \beta=2$, and the second one as $\operatorname{rank}_{1}\left(M, \operatorname{beg}_{(1+1)}+\Delta_{8}\right)=\operatorname{rank}_{1}(M, 3+1)=2$. The addition of these two values is the solution, $2+2=4$. As we can observe, the rank operation on zombit-vector only requires rank on plain bitvectors, which can be solved in $O(1)$ time by storing $o(n)$ bits of extra-space. It is important to notice that we do not discuss $\operatorname{rank}_{0}(B, i)$ because it can be solved as $i-\operatorname{rank}_{1}(B, i)$.

\section{Successor}

Given a bitvector $B$ and a position $i, \operatorname{succ}(B, i)$ returns the lowest index which contains a 1-bit at $B[i, n]$. For example, in Figure $1 \operatorname{succ}(B, 3)=3$ and $\operatorname{succ}(B, 6)=8$. This operation can be solved in the zombit-vector following Algorithm 1. Firstly, at Lines 1-2, the algorithm computes the block that contains $i\left(X_{j}\right)$, and stores the number of mixed blocks up to $X_{j}$ into $q$. There are two cases where $X_{j}$ includes the solution. The first case occurs when $X_{j} \in \mathcal{O}$, at Line 5 . Since $X_{j}$ is full of 1 s the successor is the current position $i$. The second one happens when $X_{j} \in \mathcal{M}$ and the next 1-bit is inside $X_{j}$. In order to know if the next 1-bit is contained by $X_{j}$, Line 7 computes the position $(s)$, where is the first 1-bit after the correspondent position of $i$ in $M\left(\right.$ beg $\left._{q}+\Delta_{i}\right)$. If $s$ is in the range of $X_{j}$ in $M$, [beg,$\left.e n d_{q}\right]$, the next 1-bit is in $X_{j}$. In particular, that 1-bit is at distance $\Delta_{s}$ from the first position of $X_{j}$, hence the solution is $b e g_{j}+\Delta_{s}$.

Otherwise, the next 1-bit is not in $X_{j}$ because it is empty or the last 1-bit in $X_{j}$ is previous to $i$. The algorithm jumps to the next block with 1 s by performing Algorithm 2. Firstly, the next partition $\left(X_{j^{\prime}}\right)$ that contains at least one 1-bit is computed. If it is part of $\mathcal{O}$, Line 3 , that block is full of $1 \mathrm{~s}$, and the solution is its first position. Otherwise, the result is located inside the $(q+1)$-th mixed block $\left(X_{j^{\prime}}\right)$ at position $\Delta_{s^{\prime}}$, thus the algorithm returns beg $_{j^{\prime}}+\Delta_{s^{\prime}}$.

\footnotetext{
${ }^{1}$ Note that $p^{\prime}=\operatorname{rank}_{1}(U, j-1)$ and $q^{\prime}=\operatorname{rank}_{0}(U, j-1)$.
} 


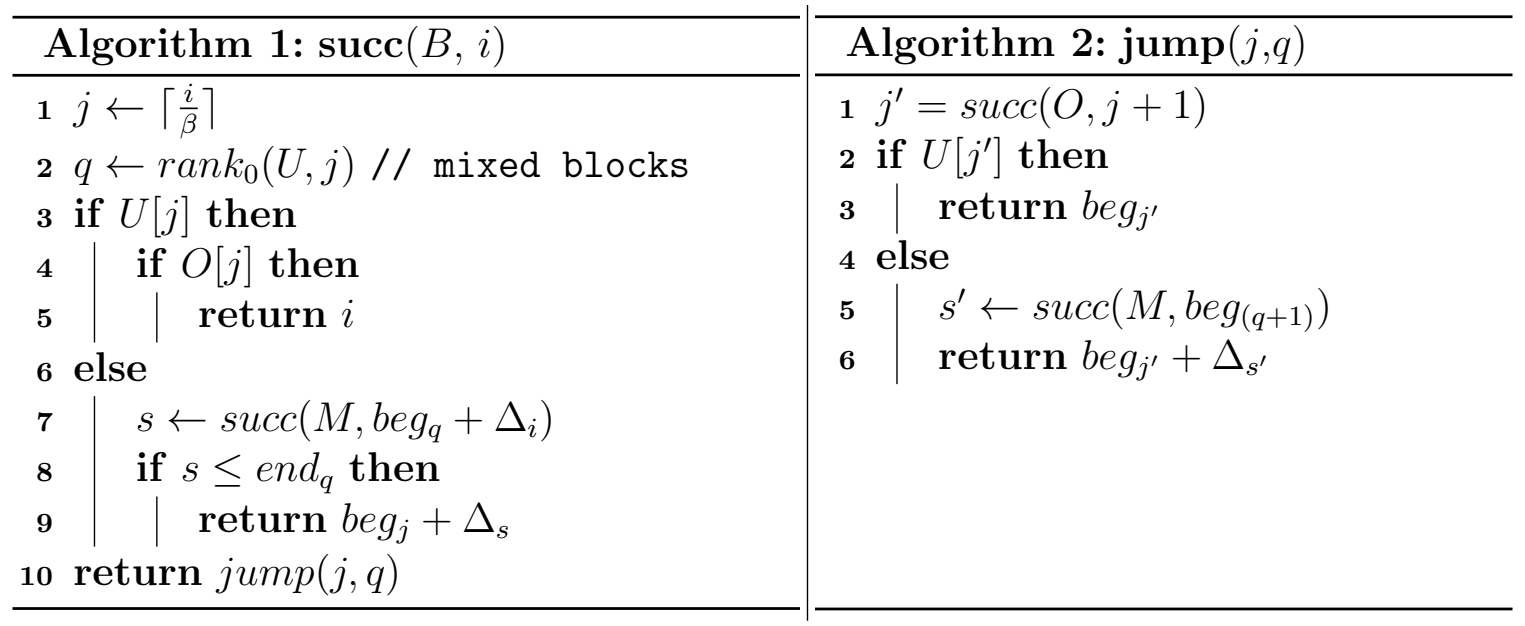

Every succ in plain bitvectors requires $o(n)$ extra space to solve them in $O(1)$ time. Consequently, zombit-vector can compute succ queries in $O(1)$ time with an extra-space of $o(n)$ bits.

Therefore access, rank, and succ can be solved in $O(1)$ time by using $O\left(k \beta+\frac{n}{\beta}\right)+$ $o(n)$ bits. With the optimal value of $\beta, \sqrt{\frac{n}{k}}$, we can reduce the space to $O(\sqrt{k n})$ bits, and keep the last operations in $O(1)$ time. Furthermore, if we apply the zombit-vector over $M$ recursively up to $c$ levels, we need to store $O\left(k^{1-\epsilon} n^{\epsilon}\right)$ bits, where $\epsilon=\frac{1}{2^{c}}$. This recursive variant converges to $O(k)$ bits, and each operation can be solved in $O\left(\log \frac{n}{k}\right)$ time. Recall that we do not discuss $\operatorname{pred}(B, i)$ because it is $\operatorname{symmetrical}$ to $\operatorname{succ}(B, i)$ and achieves identical theoretical bounds.

\section{Experimental Evaluation}

zombit-vector was coded in $\mathrm{C}++$, using several data strucures from the SDSL library [13]. We have two implementations, the basic one, zombit, and the recursive variant, zombit-rec. Both implementations compute $\beta=\sqrt{\frac{n}{k}}$, and were compared with the different proposals presented in Background (plain, $\operatorname{rrr}$ [7], rec-rank [11], sd-array [11], oz-vector [1], and hybrid-vector [12]), and Partitioned Elias-Fano [14] (pef), a technique largely used in intersection of lists. Notice that the last baseline does not support rank and access operations on bitvectors, but it gets a good compression ratio/query time trade-off in succ queries.

Taking as reference the experimental evaluation of [11], we built all the techniques over bitvectors of sizes $10^{7}, 10^{8}$, and $10^{9}$ bits with several configurations. Let us denote with run $_{0}$ (resp. run 1 ) the mean length of runs of 0s (resp. 1s), inside the input bitvector. For each dimension of bitvector, we have two distributions of runs: equal distribution, run $_{1} \simeq$ run $_{0}$, and not-equal distribution, where run $_{1} \simeq \frac{\text { run }_{0}}{8}$. In each distribution we performed various executions, with different settings for run $_{0}$ and run $_{1}$. Given the $e$-th execution, run $_{0}=10^{e}$, and the values of run $_{1}$ are computed according to the chosen distribution. Notice that, in Figure 2, we can discern which value corresponds with each execution because the size of the markers increases while run $_{0}$ grows. 


\begin{tabular}{|c|c|c|}
\hline $\begin{array}{l}--\nabla-- \text { hybrid } \\
--\Delta-- \text { oz-vector } \\
--\star-- \text { pef }\end{array}$ & $\begin{array}{l}\text {---- plain } \\
\text {--₹-- rec-rank } \\
--\square-- \text { rrr }\end{array}$ & $\begin{array}{ll}--\mathbf{- -} & \text { sd-vector } \\
--\bullet-- & \text { zombit } \\
-- \text {-- } & \text { zombit-rec }\end{array}$ \\
\hline
\end{tabular}
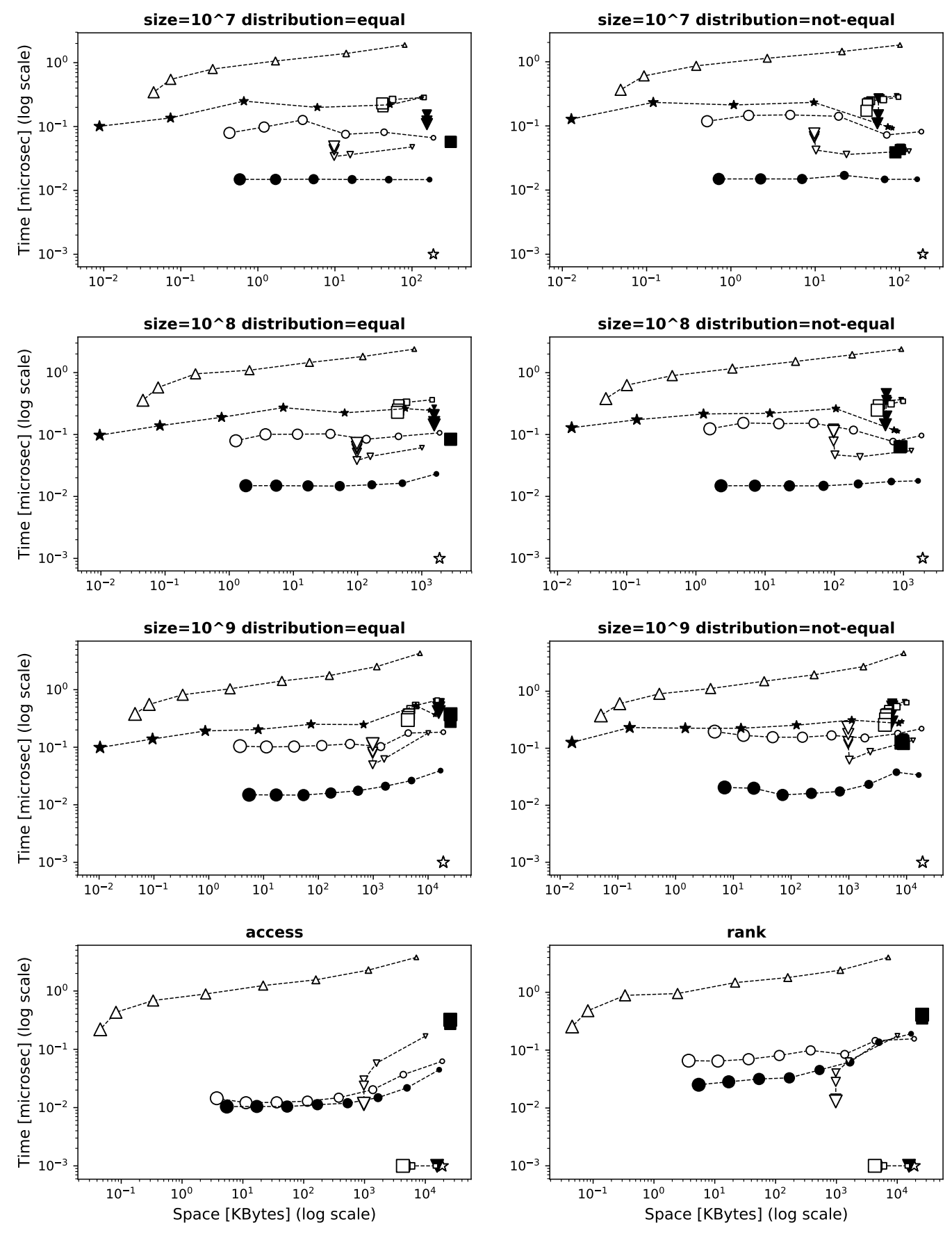

Figure 2: First six plots show the space and average response times on succ queries for different sizes and distributions of runs. The last charts show the average response times of access and rank operations on a bitvector of size $10^{9}$ with an equal distribution of runs. 
Over these data, we performed 1,000,000 random successor queries and measured the average response time per operation. In most of the baseline cases, succ was computed by using rank and select. However, some structures can solve successor more efficiently without using rank and select, specifically, sd-array and hybrid-vector. For a better comparison, in the last two structures, we have run the most efficient algorithms. The experiments were conducted on an Intel ${ }^{\circledR}$ Xeon ${ }^{\circledR}$ E5-2470 CPU (a) $2.30 \mathrm{GHz}$ (32 cores) with $20 \mathrm{MB}$ of cache and $64 \mathrm{~GB}$ of RAM, running Debian GNU/Linux 10 with kernel 4.19.0-5 (64 bits), gcc version 8.3.0 with -03.

\section{Compression}

In terms of compression, if we compare the results between the two types of distributions, there is no significant difference. The behavior is very similar except for those techniques focused on sparse bitvectors, which obtain better compression in not-equal distribution. The clear winner is pef, but it is limited in functionality. It is followed by the oz-vector, which needs $1.22 \%-48.95 \%$ of the space of zombit-rec. Concerning the rest of the techniques, when run $_{0}$ is small, the hybrid-vector is very competitive, for instance, it obtains the best compression when run $_{0}$ is lower than 10,000 in the bitvector of size $10^{9}$. However, when run $_{0}$ grows, the size of the hybrid-vector keeps constant, and it is improved by zombit, which requires $0.72 \%-54.02 \%$ of its space. Besides, we can observe that there is a slight difference between our proposal and its recursive modification, in particular, zombit-rec occupies $68.25 \%-87.06 \%$ of the space of zombit.

\section{Time performance}

As it is shown in the first six plots of Figure 2, the main competitor of our proposal for succ operations is hybrid-vector. However, it is beaten by zombit, which becomes $3-12$ times faster and keeps those times constant. Our recursive variant, which slightly improves the space, turns out 10\% - 165\% slower than hybrid-vector. Those results are similar to those obtained by rrr, sd-array, rec-rank and pef. The most timeconsuming structure is oz-vector. Though it can solve succ with the same theoretical bound of hybrid-vector, $O(\log n)$ time, in practice, it requires more binary searches, and turns 5-40 times slower than hybrid-vector.

On the bottom of Figure 2, we compare the response times of access and rank in bitvectors with size $10^{9}$ and equal distribution of runs. We can observe as zombit and zombit-rec are competitive in both operations, being close to the response times of hybrid-vector. Therefore, zombit becomes the structure with the best times in succ queries, and keeps competitive times in access and rank.

\section{Conclusions and Future Work}

We have proposed a structure, zombit, which compresses bitvectors with large runs and can solve access, rank and successor/predecessor queries in $O(1)$ time. We obtained a compression ratio of $0.04 \%-26.33 \%$, when the length of runs is larger than 100 , and we can handle successor queries $3-12$ times faster than our immediate competitor. Consequently, zombit gets a good trade-off in terms of space and time 
on bitvectors with runs. A variant of our structure to obtain better compression was introduced, but in practice, it is 5-12 times slower than zombit, and it reduces to $68.25 \%-87.06 \%$ the space of our first proposal.

As future work, since we do not beat the space of hybrid-vector in shorter runs, we will focus on improving the compression in that scenario. We plan to solve select operations on zombit efficiently by using $o(n)$ extra-space. Also, we will explore other areas where the successor and predecessor problem is relevant.

\section{References}

[1] G. Navarro, Compact Data Structures - A practical approach. Cambridge University Press, 2016.

[2] J. S. Culpepper and A. Moffat, "Efficient set intersection for inverted indexing," $A C M$ Transactions on Information Systems (TOIS), vol. 29, no. 1, p. 1, 2010.

[3] G. J. Jacobson, "Succinct static data structures," Ph.D. dissertation, Carnegie Mellon University, 1988.

[4] J. I. Munro, "Tables," in International Conference on Foundations of Software Technology and Theoretical Computer Science. Springer, 1996, pp. 37-42.

[5] D. Clark, "Compact, PAT trees," Ph.D. dissertation, University of Waterloo, Canada, 1996.

[6] R. González, S. Grabowski, V. Mäkinen, and G. Navarro, "Practical implementation of rank and select queries," in Poster Proc. Volume of 4 th Workshop on Efficient and Experimental Algorithms (WEA), 2005, pp. 27-38.

[7] R. Raman, V. Raman, and S. S. Rao, "Succinct indexable dictionaries with applications to encoding k-ary trees and multisets," in Proceedings of the thirteenth annual ACM-SIAM symposium on Discrete algorithms. Society for Industrial and Applied Mathematics, 2002, pp. 233-242.

[8] R. Pagh, "Low redundancy in static dictionaries with o (1) worst case lookup time," in International Colloquium on Automata, Languages, and Programming. Springer, 1999, pp. 595-604.

[9] A. Golynski, "Optimal lower bounds for rank and select indexes," Theoretical Computer Science, vol. 387, no. 3, pp. 348-359, 2007.

[10] P. B. Miltersen, "Lower bounds on the size of selection and rank indexes," in Proceedings of the sixteenth annual ACM-SIAM symposium on Discrete algorithms. Society for Industrial and Applied Mathematics, 2005, pp. 11-12.

[11] D. Okanohara and K. Sadakane, "Practical entropy-compressed rank/select dictionary," in Proceedings of the Meeting on Algorithm Engineering $\& 5$ Expermiments. Society for Industrial and Applied Mathematics, 2007, pp. 60-70.

[12] J. Kärkkäinen, D. Kempa, and S. J. Puglisi, "Hybrid compression of bitvectors for the FM-index," in 2014 Data Compression Conference. IEEE, 2014, pp. 302-311.

[13] S. Gog, T. Beller, A. Moffat, and M. Petri, "From theory to practice: Plug and play with succinct data structures," in Proc. 13th International Symposium on Experimental Algorithms (SEA), 2014, pp. 326-337.

[14] G. Ottaviano and R. Venturini, "Partitioned elias-fano indexes," in Proceedings of the 37th international ACM SIGIR conference on Research 85 development in information retrieval. ACM, 2014, pp. 273-282. 